\title{
Monitoring the localization-delocalization transition within a one-dimensional model with nonrandom long-range interaction
}

\author{
A. V. Malyshev* \\ Departamento de Física Aplicada, Universidad de Salamanca, E-37071 Salamanca, Spain \\ V. A. Malyshev ${ }^{\dagger}$ \\ Institute for Theoretical Physics and Materials Science Center, University of Groningen, \\ Nijenborgh 4, 9747 AG Groningen, The Netherlands
}

F. Domínguez-Adame

Grupo Interdisciplinar de Sistemas Complejos, Departamento de Física de Materiales, Universidad Complutense, E-28040 Madrid, Spain

(Received 5 March 2003; revised manuscript received 15 June 2004; published 9 November 2004)

\begin{abstract}
We consider a two-parameter one-dimensional Hamiltonian with uncorrelated diagonal disorder and nonrandom long-range intersite interaction $J_{m n}=J /|m-n|^{\mu}$. The model is critical at $1<\mu<3 / 2$ and reveals the localization-delocalization transition with respect to the disorder magnitude. To detect the transition we analyze level and wave function statistics. It is demonstrated also that in the marginal case $(\mu=3 / 2)$ all states are localized.
\end{abstract}

DOI: 10.1103/PhysRevB.70.172202

The localization-delocalization transition (LDT) in disordered systems, predicted by Anderson for three dimensions in $1958,{ }^{1}$ (see also Ref. 2) still remains a fascinating problem (see Refs. 3-5 for an overview). During the last two decades, remarkable progress has been achieved in understanding the LDT, especially in discovering the nature of wave functions at transition. This progress became possible thanks to the fruitful idea of the multifractality of wave functions at criticality. ${ }^{6-10}$ This conjecture was analytically proven for an ensemble of power-law random banded matrices (PRMB), which revealed the LDT with respect to the interaction exponent $^{11,12}$ (see Ref. 5 for an overview). Within the framework of the latter model, it was demonstrated, in particular, that (i) the distribution function of the inverse participation ratio (IPR) is scale invariant at transition and (ii) the relative IPR fluctuation (the ratio of the standard deviation to the mean) is of the order of unity at the critical point. ${ }^{13,14}$ This finding confirmed the conjecture that was put forward for the first time in Refs. 15 and 16 that distributions of relevant physical magnitudes are universal at criticality (see also Refs. 17-19). This invariance is a powerful tool to monitor the critical point.

In the present paper, we consider a two-parameter tightbinding Hamiltonian on a regular one-dimensional (1D) lattice of size $N$ with nonrandom long-range intersite interaction:

$$
\mathcal{H}=\sum_{n=1}^{N} \varepsilon_{n}|n\rangle\left\langle n\left|+\sum_{m, n=1}^{N} J_{m n}\right| m\right\rangle\langle n|,
$$

where $|n\rangle$ is the ket vector of a state with on-site energy $\varepsilon_{n}$. These energies are stochastic variables, uncorrelated for different sites and distributed uniformly around zero within the interval of width $\Delta$. The hopping integrals are $J_{m n}=J / \mid m$ $-\left.n\right|^{\mu}, J_{n n}=0$ with $1<\mu \leqslant 3 / 2$. For definiteness we set $J>0$, then the LDT with respect to disorder magnitude occurs at the upper band edge, provided $1<\mu<3 / 2 .^{20,21}$ The transi-
PACS number(s): 71.30.+h, 72.15.Rn, 78.30.Ly, 36.20.Kd

tion is similar to that within the standard 3D Anderson model. $\mu=3 / 2$ represents the marginal case in which all states are expected to be weakly localized. ${ }^{21}$

To detect the transition we analyze level and wave function statistics. We perform a numerical analysis of size and disorder scaling of the relative fluctuation of both the nearest-level spacing (LS) and the participation number (PN). The latter is defined as

$$
P_{\nu}=\left[\sum_{n=1}^{N}\left|\psi_{\nu n}\right|^{4}\right]^{-1},
$$

where $\psi_{\nu n}$ denotes the $n$th component of the $\nu$ th normalized eigenstate of the Hamiltonian (1).

The relative fluctuation of the nearest-level spacing is an invariant parameter at transition, as was conjectured in Ref. 17 for the 3D Anderson model and demonstrated later for a variety of other disordered models (see, e.g., Refs. 5 and references therein). The invariance can be used to detect the critical point. We demonstrate that within the present model, the ratio of the standard deviation of the PN (SDPN) to its mean value (MPN) is also an invariant parameter at the critical disorder magnitude $\Delta_{c}$. Therefore, the ratio SDPN/MPN can also be used to detect the transition. To the best of our knowledge, this quantity has never been used for this purpose.

As the LDT occurs at the top of the band within the considered two-parameter model, we calculate disorder and size scaling for uppermost states. Open chains are used in all calculations. We take advantage of the Lanczos method to calculate the scaling for large system sizes (up to about 6 $\times 10^{4}$ sites) and two particular values of the interaction exponent: $\mu=4 / 3$ (the LDT occurs) and $\mu=3 / 2$ (the marginal case; no transition is expected ${ }^{21}$ ).

First, we calculate the critical point by means of the level statistics analysis. In Fig. 1 we plotted the disorder scaling of the ratio of the standard deviation of the nearest-level spac- 


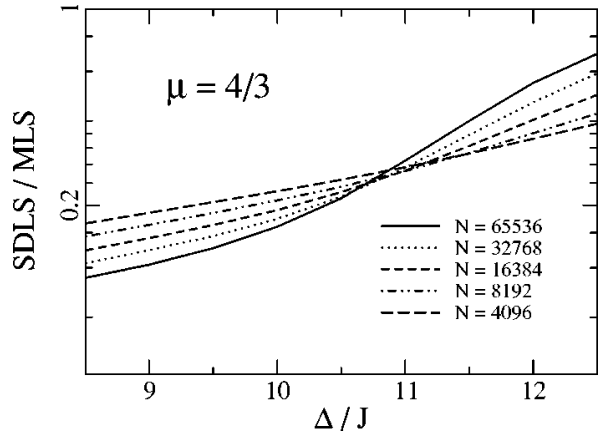

FIG. 1. Disorder scaling of the relative fluctuation of the nearest-level spacing (the ratio SDLS/MLS) for $\mu=4 / 3$ in the vicinity of the joint intersection point [that is at $\Delta=(10.7-11.5) J$ ]. The curves are calculated for different system sizes $N$ and averaged over more than $5 \times 10^{3} \times(65536 / N)$ disorder realizations.

ing (SDLS) distribution to its mean (MLS) at the top of the band for $\mu=4 / 3$. The figure demonstrates that all disorderscaling curves plotted for different system sizes intersect within a narrow range of $\Delta$, between $10.7 \mathrm{~J}$ and $11.5 \mathrm{~J}$.

Calculations of the scaling of the relative PN fluctuation confirm the conjecture that the ratio SDPN/MPN is also a size invariant parameter at transition: Figure 2 shows that all SDPN/MPN curves plotted versus disorder for different system sizes intersect in a narrow range of $\Delta$, from $10.0 \mathrm{~J}$ to 10.6J. One can deduce from Fig. 2 that both the MPN and the SDPN are of the same order of magnitude at the intersection for any system size, as was shown for other models in Refs. 11, 13, 14, 18, and 19.

The regular size dependence of intersection points in Figs. 1 and 2 is a finite size effect; accounting for the latter by means of the finite size scaling analysis allows for obtaining the value of the critical disorder. Both figures demonstrate that finite size effects are unusually strong, which, within the present model, results from the long-range nature of the intersite interaction. Contrary to the standard Anderson model, the contribution of the long-range coupling terms to the spectrum of the Hamiltonian (1) converges very slowly as the system size increases. The latter results in a corresponding increase of the bandwidth (mostly, the upper band edge,

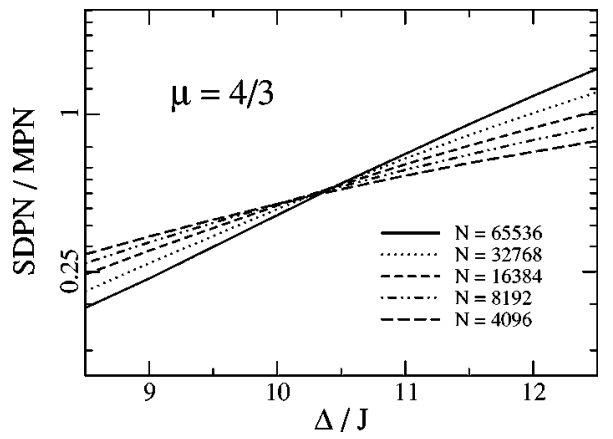

FIG. 2. Disorder scaling of the relative fluctuation of the PN (the ratio SDPN/MPN) for $\mu=4 / 3$ in the vicinity of the joint intersection point [that is at $\Delta=(10.0-10.6) J$ ]. The curves are calculated for different system sizes $N$ and averaged over more than $5 \times 10^{3}$ $\times(65536 / N)$ disorder realizations. where the LDT takes place). For an open chain, the upper band edge $E(N)$ scales in size as follows:

$$
E(N)=E_{\infty}(\mu)-\frac{C(\mu)}{N^{\mu-1}}+O\left(N^{-\mu}\right) .
$$

For $\mu=4 / 3, E_{\infty}(4 / 3) \approx 7.20 J$ and $C(4 / 3) \approx 8.45 J$. The increase of the bandwidth with the system size leads to the fact that disorder of the same magnitude is effectively weaker for larger systems. The latter effect introduces regular size dependence of the critical disorder that is obtained by numerical analyses of finite systems. The contribution of other finite size effects, ${ }^{22,23}$ such as influence of boundary regions, are expected to be weaker for large systems because of very slow convergence of the upper band edge $\left(\propto N^{1-\mu}\right)$. Our calculations confirm this conjecture.

The intersection point, $\Delta\left(N_{1}, N_{2}\right)$, of two disorder scaling curves plotted for different system sizes $N_{1}$ and $N_{2}$ depends on the sizes. To account for such dependencies we proceed as follows. First, set by definition:

$$
\Delta_{c}(N)=\Delta(N-1, N+1), N_{r} \rightarrow \infty,
$$

where $\Delta_{c}(N)$ is the critical disorder that can be obtained by analyses of a finite system of size $N\left(N_{r}\right.$ is the number of disorder realizations over which the averaging is performed). Second, use the following ansatz for the intersection point:

$$
\Delta\left(N_{1}, N_{2}\right)=w\left(N_{1}\right) \Delta_{c}\left(N_{1}\right)+w\left(N_{2}\right) \Delta_{c}\left(N_{2}\right),
$$

where the weight function $w(N)$ is to be determined. Bearing in mind the slow convergence of the band edge $\left(\propto N^{1-\mu}\right)$, we use the following ansatz for $\Delta_{c}(N)$ :

$$
\Delta_{c}(N) \approx \Delta_{c}(\infty)+b N^{1-\mu}+c N^{-\gamma}, N \gg 1
$$

where the $b, c$, and $\gamma>\mu-1$ are fitting parameters. Using the ansatz (6) together with Eqs. (4) and (5) and expanding $\Delta(N-1, N+1)$ in series about $N$ (at $N \gg 1$ ), we find the weight function: $w(N)=1 / 2+O\left(1 / N^{\mu+1}\right)$. Further, for any given pair $N_{1}<N_{2}\left(N_{1}, N_{2} \gg 1\right)$ there exists the size $N$, such that $\Delta_{c}(N)=\Delta\left(N_{1}, N_{2}\right)$. Making use of the latter equation together with Eq. (6) and keeping the leading (nonzero) power of system size in all expansions, we find the sought $N$ :

$$
N=\frac{2^{1 / p} N_{1} N_{2}}{\left(N_{1}^{p}+N_{2}^{p}\right)^{1 / p}}, p=\mu-1 .
$$

Thus, the intersection point of disorder-scaling curves plotted for two different system sizes $N_{1}<N_{2}\left(N_{1}, N_{2} \gg 1\right)$ yields the critical disorder for an intermediate system size $N$ as defined by Eq. (7).

We further use Eq. (7) and intersection points of the curves in Fig. 1 (LS data) and Fig. 2 (PN data) to obtain $\Delta_{c}(N)$. Figure 3 shows $\Delta_{c}(N)$ together with best nonlinear fits of Eq. (6) to the whole data sets (dashed lines) and the best linear fit of $E(N)$ given by Eq. (3) to the three last PN-data points (solid line). The nonlinear fits give $\Delta_{c}(\infty)$ $=(10.97 \pm 0.09) \mathrm{J}$ for the LS data, and $\Delta_{c}(\infty)$ $=(11.19 \pm 0.10) J$ for the whole PN data set, while the linear fit of $E(N)$ to the tail PN points gives $\Delta_{c}(\infty)$ $=(10.91 \pm 0.17) J$. The obtained values of $\Delta_{c}(\infty)$ agree well 


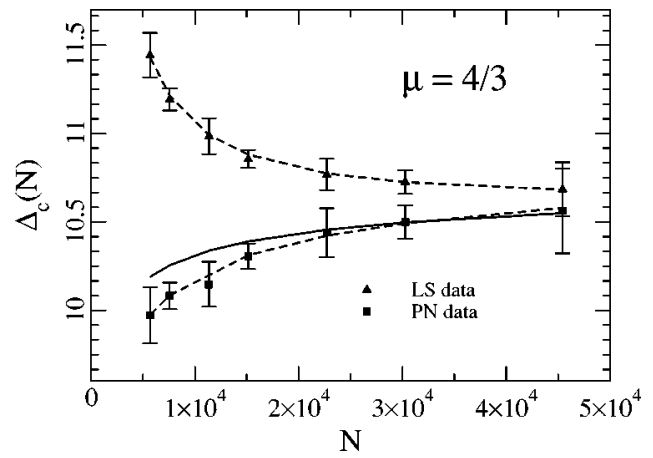

FIG. 3. Critical disorder sizes scaling obtained from the PN data $\boldsymbol{\square})$ and level statistics data $(\boldsymbol{\Delta})$. Dashed lines are best fits of Eq. (6) to the LS data $(b=-26.44, c=824.16, \gamma=0.70)$ and the PN data $(b=-21.61, c=12.73, \gamma=2.82)$. The solid line is the best linear fit of Eq. (3) to the last three PN data points: $\Delta_{c}(N)$ $=(1.52 \pm 0.02) \times E(N)$.

with each other. This confirms our conjecture that for large system sizes the band edge size dependence provides the dominant contribution to the finite size effects. Finally, the critical disorder is determined as $\Delta_{c}(\infty)=(11.09 \pm 0.21) J$ for $\mu=4 / 3$.

It should be noticed that, despite that both methods to detect the LDT are in good agreement, finite size effects are more pronounced in the case of analysis of the level statistics. For these reasons, the proposed method which is based on the studies of the wave function statistics appears to be advantageous, at least for the considered model.

We applied the same technique to analyze the localization properties in the marginal case, $\mu=3 / 2$, where the states are expected to be localized weakly. ${ }^{21}$ Figure 4 shows the SDPN/ MPN scaling curves in the vicinity of the only joint intersection point that appears to be trivial: $\Delta_{c}=0$. Size scaling of the ratio MPN/N (see Fig. 5) reveals no transition too; all MPN/N size-scaling curves for nonzero magnitude of disorder decrease with system size, as they do for a localized (or critical) state. Thus, no signatures of the LDT can be observed in the marginal case, indicating that all states are localized.

In summary, we studied numerically the critical properties of the 1D two-parameter tight-binding model with diagonal disorder and nonrandom long-range interaction, $J_{m n}=J / \mid m$ $-\left.n\right|^{\mu}, J>0$ and $1<\mu \leqslant 3 / 2$. The transition point was detected by means of the level and wave function statistics. We used the conjecture on the scale invariance of the distribution function of the nearest-level spacing and the participation number at criticality. We find, in particular, that the critical point for $\mu=4 / 3$ is $\Delta_{c}=(11.09 \pm 0.21) J$. In the marginal case $(\mu=3 / 2)$, that is analogous to the standard 2D Anderson model, ${ }^{21}$ the only joint intersection point is $\Delta_{c}=0$, indicating that all states are localized for a finite disorder.

We demonstrated that finite size effects are very pronounced within the considered model. Level statistics appears to be more affected by these effects as compared to the participation number statistics. The dominant contribution to finite size effects is determined by the size dependence of the bandwidth. To obtain the critial disorder, we use a reformulated finite size scaling procedure that is corrected for irrelevant size dependencies.

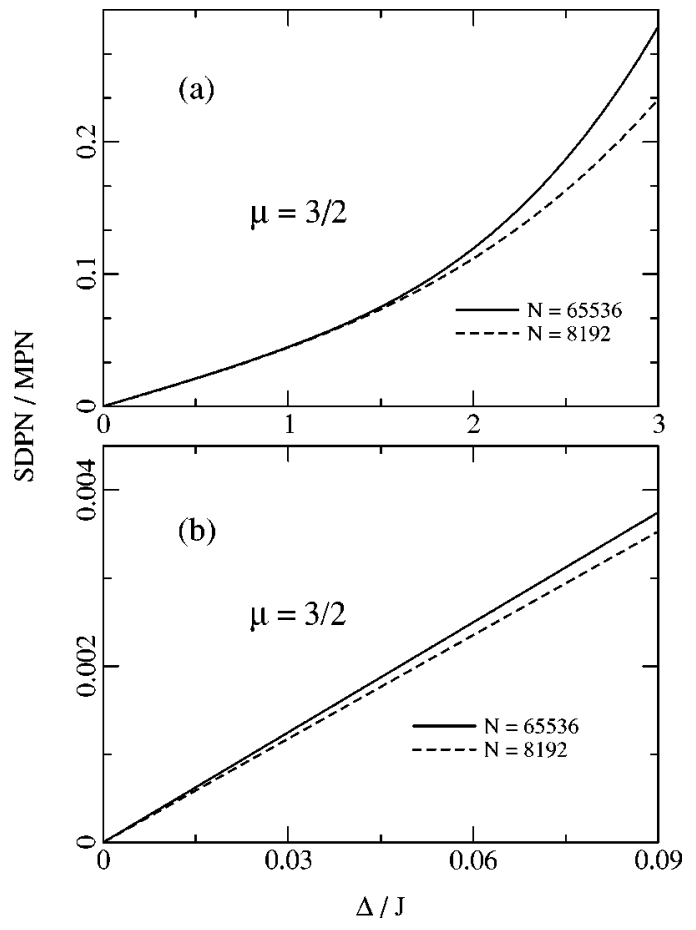

FIG. 4. (a) Disorder scaling of the relative PN fluctuation (the ratio SDPN/MPN) for $\mu=3 / 2$ in the vicinity of the joint intersection point at $\Delta_{c}=0$. The curves are calculated for two different system sizes (65536 and 8192) and averaged over more than 5 $\times 10^{3}$ and $10^{5}$ disorder realizations, respectively. (b) An enlargement of the crossing at the origin.

To conclude, we stress that the scale invariance of the relative fluctuation of the participation number at transition is a consequence of critical wave function fluctuations. We conjecture, therefore, that the analysis of the relative fluctuation of the participation number provides a general tool to monitor the LDT. The proposed method proves to work well for the standard 3D Anderson model too. ${ }^{24}$ We believe also that this property holds at the mobility edge, allowing therefore to monitor the latter.

The authors thank A. Rodríguez, M. A. Martín-Delgado, and G. Sierra for discussions. This work was supported by DGI-MCyT (MAT2003-01533) and MECyD (SB20010146).

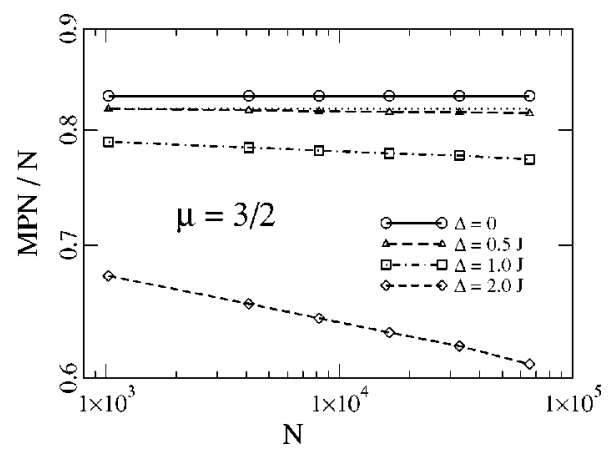

FIG. 5. Size scaling of the ratio MPN/N calculated for $\mu=3 / 2$ and different disorder magnitudes. Thin horizontal dotted line is a guide for the eye. 
*On leave from Ioffe Physiko-Technical Institute, 26 Politechnicheskaya str., 194021 Saint-Petersburg, Russia.

'On leave from “S.I. Vavilov State Optical Institute," 199034 SaintPetersburg, Russia.

${ }^{1}$ P. W. Anderson, Phys. Rev. 109, 1492 (1958).

${ }^{2}$ E. Abrahams, P. W. Anderson, D. C. Licciardello, and T. V. Ramakrishnan, Phys. Rev. Lett. 42, 673 (1979).

${ }^{3}$ P. A. Lee and T. V. Ramakrishnan, Rev. Mod. Phys. 57, 287 (1985).

${ }^{4}$ B. Kramer and A. MacKinnon, Rep. Prog. Phys. 56, 1469 (1993).

${ }^{5}$ A. D. Mirlin, Phys. Rep. 326, 259 (2000).

${ }^{6}$ F. Wegner, Z. Phys. B 36, 209 (1980); Nucl. Phys. B 316, 663 (1989).

${ }^{7}$ H. Aoki, J. Phys. C 16, L205 (1983); Phys. Rev. B 33, 7310 (1986).

${ }^{8}$ C. Castellani and L. Peliti, J. Phys. A 19, L429 (1986).

${ }^{9}$ M. Schreiber and H. Grussbach, Phys. Rev. Lett. 67, 607 (1991); Mod. Phys. Lett. B 6, 851 (1992).

${ }^{10}$ M. Janssen, Int. J. Mod. Phys. B 8, 943 (1994).

${ }^{11}$ A. D. Mirlin, Y. V. Fyodorov, F.-M. Dittes, J. Quezada, and T. H. Seligman, Phys. Rev. E 54, 3221 (1996).

${ }^{12}$ L. S. Levitov, Europhys. Lett. 9, 83 (1989); Ann. Phys. (Leipzig)
8, 507 (1999).

${ }^{13}$ F. Evers and A. D. Mirlin, Phys. Rev. Lett. 84, 3690 (2000).

${ }^{14}$ A. D. Mirlin and F. Evers, Phys. Rev. B 62, 7920 (2000).

${ }^{15}$ B. Shapiro, Phys. Rev. B 34, 4394 (1986); Philos. Mag. B 56, 1031 (1987).

${ }^{16}$ A. Cohen, Y. Roth, and B. Shapiro, Phys. Rev. B 38, 12125 (1988).

${ }^{17}$ B. I. Shklovskii, B. Shapiro, B. R. Sears, P. Lambrianides, and H. B. Shore, Phys. Rev. B 47, 11487 (1993).

${ }^{18}$ Y. V. Fyodorov and A. D. Mirlin, Phys. Rev. B 51, 13403 (1995).

${ }^{19}$ V. N. Prigodin and B. L. Altshuler, Phys. Rev. Lett. 80, 1944 (1998).

${ }^{20}$ A. Rodríguez, V. A. Malyshev, and F. Domínguez-Adame, J. Phys. A 33, L161 (2000).

${ }^{21}$ A. Rodríguez, V. A. Malyshev, G. Sierra, M. A. Martín-Delgado, J. Rodríguez-Laguna, and F. Domínguez-Adame, Phys. Rev. Lett. 90, 027404 (2003).

${ }^{22}$ A. MacKinnon, J. Phys.: Condens. Matter 6, 2511 (1994)

${ }^{23}$ K. Slevin and T. Ohtsuki, Phys. Rev. Lett. 82, 382 (1999)

${ }^{24}$ G. Schubert, A. Weisse, and H. Fehske, cond-mat/0309015 (unpublished), private communication. 